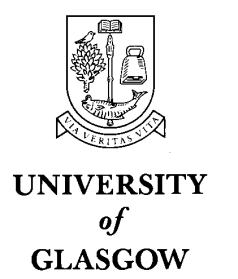

Department of Physics \& Astronomy Experimental Particle Physics Group

Kelvin Building, University of Glasgow,

Glasgow, G12 8QQ, Scotland

GLASGOW Telephone: +44 (0)1413398855 Fax: +44 (0)1413305881
GLAS-PPE/97-08

October 1997

\title{
Hadronic Final States in Deep Inelastic Scattering at HERA
}

\author{
N. H. Brook \\ Lecture given at "The Actual Problems of Particle Physics", Gomel, Belarus.
}

\begin{abstract}
This lecture contains a brief introduction to HERA and deep inelastic scattering (DIS), before going on to highlight some of the measurements of the hadronic final state in DIS performed by the $\mathrm{H} 1$ and ZEUS collaborations.
\end{abstract}




\section{The HERA Accelerator and Detectors}

The HERA accelerator, located at DESY in Hamburg, is an electron-proton collider. It is $6.3 \mathrm{~km}$ in circumference and collides positrons (or electrons) at $\approx 27 \mathrm{GeV}$ with protons at $820 \mathrm{GeV}$. There are four interaction regions: two containing general purpose, hermetic detectors (H1 and ZEUS); another experiment (HERMES) investigating the spin distributions of the quarks in protons and neutrons; and another (HERA-B) planning to measure CP violation in the B-system. The H1 and ZEUS detectors took first data in 1992.

The ZEUS detector is shown in figure 1. The asymmetric design of the detector reflects the proton energy being significantly higher than that of the electron beam.

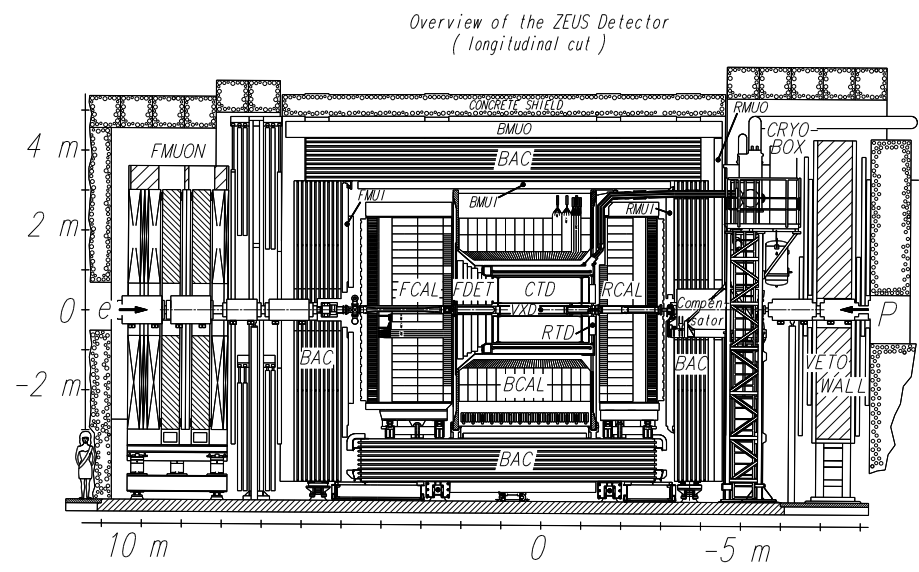

Figure 1: Cross sectional view of the ZEUS detector

The tracking system consists of a vertex detector (VXD) [1] and a central tracking chamber (CTD) [2] enclosed in a $1.43 \mathrm{~T}$ solenoidal magnetic field. Immediately surrounding the beampipe is the VXD which consists of 120 radial cells, each with 12 sense wires. The CTD, which encloses the VXD, is a drift chamber consisting of 72 cylindrical layers, arranged in 9 superlayers. Superlayers with wires parallel to the beam axis alternate with those inclined at a small angle to give a stereo view. A forward tracking detector is employed in the forward region to detect tracks in the proton direction and consists of three 12-layer planar drift chambers sandwiched with pairs of transition radiation detectors. In the rear direction there is an additional 12-layer planar drift chamber known as the rear tracking detector (RTD).

Outside the solenoid is the uranium-scintillator calorimeter (CAL) [3], which is divided into three parts: forward, barrel and rear covering the polar regions $2.6^{\circ}$ to $36.7^{\circ}, 36.7^{\circ}$ to $129.1^{\circ}$ and $129.1^{\circ}$ to $176.2^{\circ}$, respectively. The CAL covers $99.7 \%$ of the solid angle, with holes of $20 \times 20$ $\mathrm{cm}^{2}$ in the centres of the forward and rear calorimeters to accommodate the HERA beam pipe. Each of the calorimeter parts is subdivided into towers which are segmented longitudinally into electromagnetic (EMC) and hadronic (HAC) sections. The small angle rear tracking detector (SRTD) [4], which is attached to the front face of the rear calorimeter, measures the impact point of charged particles at small angles with respect to the positron beam direction.

The iron return yoke for the magnet is instrumented with proportional counters. This backing calorimeter (BAC) measures any hadronic energy which 'leaks out' out of the main calorimeter. Beyond that and in the forward direction there are further detectors for muon detection.

Downstream of the main detector in the proton direction, six measuring stations are installed in the proton ring for detecting forward scattered protons. Beyond the final station, further downstream, is a forward neutron calorimeter. In the electron direction, two lead scintillator 
calorimeters placed $-35 \mathrm{~m}$ and $-107 \mathrm{~m}$ from the interaction point measure the luminosity and tag events with a small momentum transfer [5].

A fuller description of the ZEUS detector can be found in reference [6]. The H1 detector is of a very similar layout as ZEUS and a description can be found in reference [7].

\section{DIS Kinematics}

The event kinematics of deep inelastic scattering, DIS, are determined by the negative square of the four-momentum transfer at the positron vertex, $Q^{2} \equiv-q^{2}$, and the Bjorken scaling variable, $x=Q^{2} / 2 P \cdot q$, where $P$ is the four-momentum of the proton. In the Quark Parton Model (QPM), the interacting quark from the proton carries the four-momentum $x P$. The variable $y$, the fractional energy transfer to the proton in its rest frame, is related to $x$ and $Q^{2}$ by $y \simeq Q^{2} / x s$, where $\sqrt{s}$ is the positron-proton centre of mass energy. Because the H1 and ZEUS detectors are almost hermetic the kinematic variables $x$ and $Q^{2}$ can be reconstructed in a variety of ways using combinations of electron and hadronic system energies and angles [8].

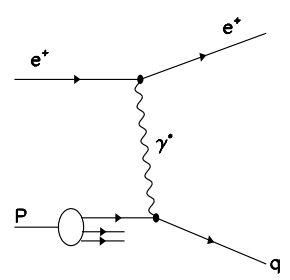

(a)

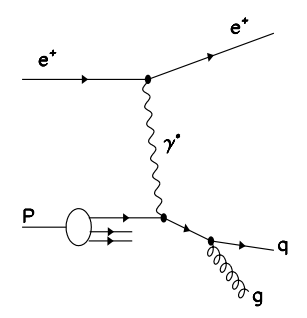

(b)

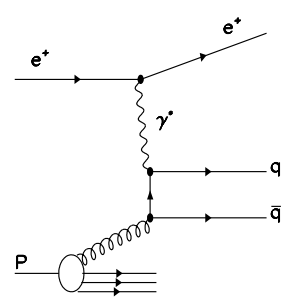

(c)

Figure 2: (a) QPM (b) QCDC and (c) BGF diagrams

In QPM there is a 1+1 parton configuration, fig. 2a, which consists of a single struck quark and the proton remnant, denoted by "+1". At HERA energies there are significant higherorder Quantum Chromodynamic (QCD) corrections: to leading order in the strong coupling constant, $\alpha_{\mathrm{s}}$, these are QCD-Compton scattering (QCDC), where a gluon is radiated by the scattered quark and Boson-Gluon-Fusion (BGF), where the virtual boson and a gluon fuse to form a quark-antiquark pair. Both processes have $2+1$ partons in the final state, as shown in fig. 2. There also exists calculations for the higher, next-to-leading (NLO) processes.

Perturbative QCD does not predict the absolute value of the parton densities within the proton but determines how they vary from a given input. For a given initial distribution at a particular scale Altarelli-Parisi (DGLAP) evolution [9] enables the distributions at higher $Q^{2}$ to be determined. DGLAP evolution resums the leading $\log \left(Q^{2}\right)$ contributions associated with a chain of gluon emissions. At large enough electron-proton centre-of-mass energies there is a second large variable $1 / x$ and, therefore, it is also necessary to resum the $\log (1 / x)$ contributions. This is acheived by using the BFKL equation [10].

\section{Jet Physics}

To relate the hadronic final state to the underlying hard partonic behaviour it is generally necessary to apply a jet algorithm. The JADE algorithm [11] has been used in the following analyses as it was, at the time, the only algorithm which allowed comparison to the NLO calculations (PROJET [12 and DISJET [13). The JADE algorithm is a cluster algorithm 
based on the scaled invariant mass-squared

$$
y_{i j}^{\mathrm{JADE}}=\frac{2 E_{i} E_{j}\left(1-\cos \theta_{i j}\right)}{W^{2}}
$$

for any two objects $i$ and $j$ assuming that these objects are massless. $W^{2}$ is the squared invariant mass of the hadronic final state and $\theta_{i j}$ is the angle between the two objects of energies $E_{i}$ and $E_{j}$. The minimum $y_{i j}$ of all possible combinations is found. If the value of this minimum $y_{i j}$ is less than the variable cut-off parameter $y_{c u t}$, the two objects $i$ and $j$ are merged into a new object by adding their four-momenta and the process is repeated until all $y_{i j}>y_{\text {cut }}$. The surviving objects are called jets which represent the underlying partonic structure that is dependent on $\alpha_{s}$.

\section{ZEUS 1994}
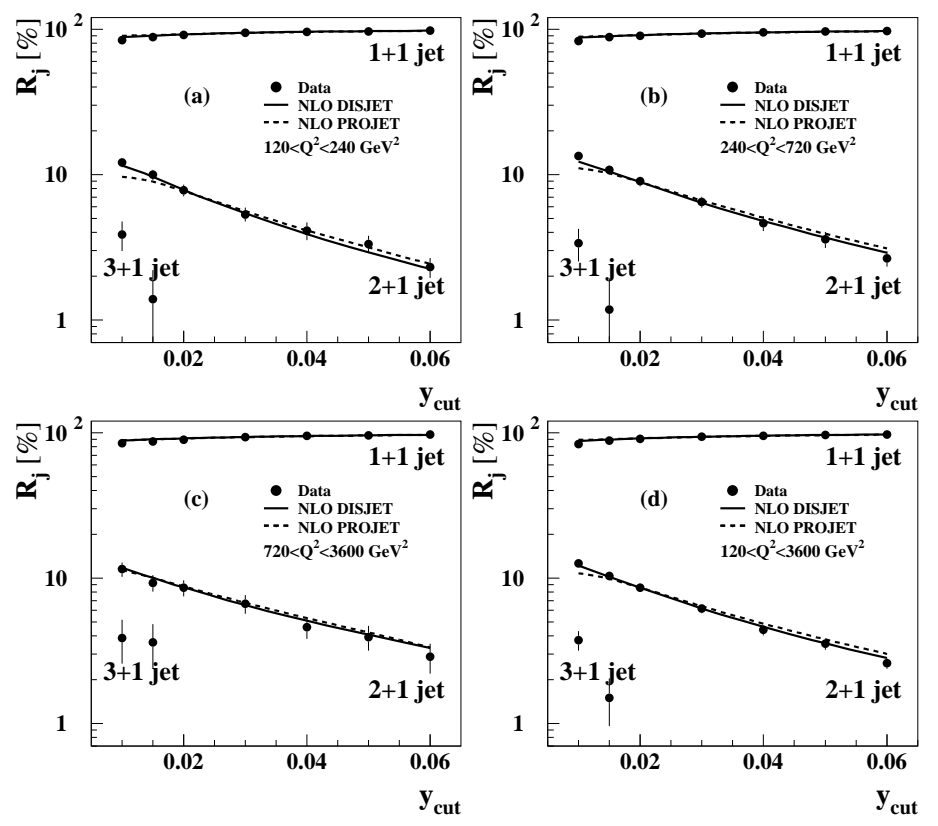

Figure 3: Jet production rates $R_{j}$ as a function of the jet resolution parameter $y_{\text {cut }}$ for $Q^{2}$ in the range (a) $120<Q^{2}<240 \mathrm{GeV}^{2}$, (b) $240<Q^{2}<720 \mathrm{GeV}^{2}$, (c) $720<Q^{2}<3600 \mathrm{GeV}^{2}$, and (d) $120<Q^{2}<3600 \mathrm{GeV}^{2}$. Only statistical errors are shown. Two NLO QCD calculations, DISJET and PROJET, each with the value of $\Lambda_{\overline{M S}}$ obtained from the fit at $y_{\text {cut }}=0.02$, are also shown.

Figures 3 a-d show the ZEUS jet rates using data taken in $1994, R_{1+1}, R_{2+1}$ and $R_{3+1}$ as a function of $y_{\text {cut }}$ for data compared with the DISJET and PROJET NLO QCD calculations for three $Q^{2}$ intervals $120<Q^{2}<240 \mathrm{GeV}^{2}, 240<Q^{2}<720 \mathrm{GeV}^{2}, 720<Q^{2}<3600 \mathrm{GeV}^{2}$, and the combined region $120<Q^{2}<3600 \mathrm{GeV}^{2}$. There is good agreement between the corrected $1+1$ and $2+1$ jet rates and the NLO QCD calculation over most of the range in $y_{\text {cut }}$ shown. Both programs agree well in their prediction of the jet-rate dependence as a function of $y_{c u t}$.

The values of $\alpha_{s}(Q)$ extracted by the H1 [14 and ZEUS [15] collaboration as a function of $Q$ are shown in Fig. 4 . The value of $\alpha_{s}$ was determined by varying the $\Lambda$ scale parameter in the QCD calculation until the best fit to the ratio $R_{2+1}$ was obtained at a particular value of $y_{\text {cut }}$. The measured $\alpha_{s}$ decreases with increasing $Q$, consistent with the running of the strong coupling constant, with $Q^{2}$ taken as the scale. In addition the figure shows the curves for $\Lambda \frac{(5)}{M S}$ 

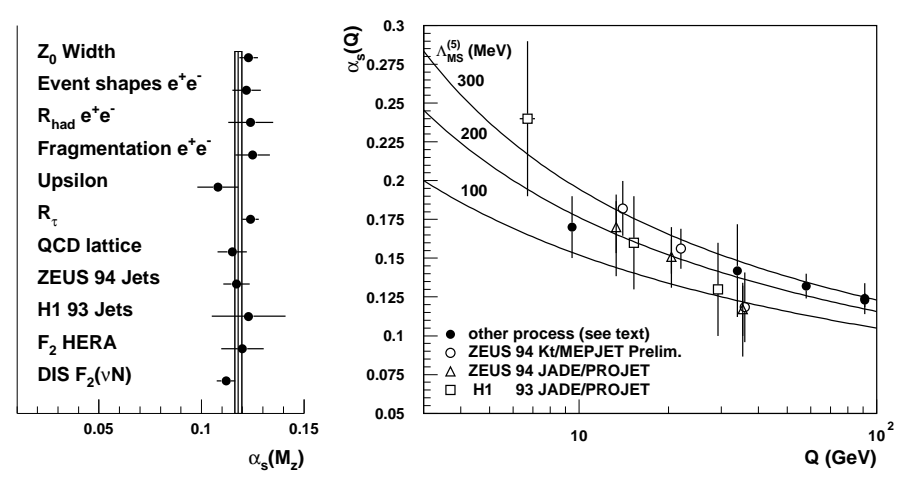

Figure 4: Left: Values and total error of $\alpha_{s}\left(M_{Z}\right)$ from various processes. The solid line indicates the world average and the band its total error. Right: $\alpha_{s}(Q)$ from HERA (open symbols) and other processes with increasing $Q$ (closed circles): $\Gamma_{\Upsilon}$ and $\sigma_{\text {had }} / \sigma_{\text {tot }}$, event shapes and $\Gamma_{\text {hadron }} / \Gamma_{\text {lepton }}$ in $e^{+} e^{-}$.

$=100,200$, and $300 \mathrm{MeV}$. An extrapolation to $\alpha_{s}\left(M_{z}\right)$ yields:

$$
\begin{gathered}
\text { H1 } 93: \quad \alpha_{s}\left(M_{z}\right)=0.123 \pm 0.012(\text { stat }) \pm 0.013 \text { (syst.) } \\
\text { ZEUS } 94: \alpha_{s}\left(M_{z}\right)=0.117 \pm 0.005(\text { stat })_{-0.004}^{+0.005}(\text { exp. }) \pm 0.007 \text { (th.) }
\end{gathered}
$$

which are consistent with other values obtained from a large variety of different processes as shown Fig. \(see [16] for references). Even with the current statistics the HERA measurements are already competitive with those made elsewhere.

Recently two new, more flexible NLO calculations (MEPJET [17 and DISENT [18) have become available allowing the experiments to analyze the data using any particular jet algorithm. The $k_{T}$ algorithm [19] is particularly suited for DIS as it allows factorization between the beam fragmentation and the hard process [20]. The ZEUS collaboration has reanalyzed [21] their 1994 data using this algorithm. The preliminary values of $\alpha_{s}(Q)$ obtained in the three bins of $Q$ are shown (with statistical errors only) in Fig. 1 and are consistent with the results obtained with the JADE algorithm.

\section{Event Shapes}

A natural frame in which to study the dynamics of the hadronic final state in DIS is the Breit frame [22]. In this frame the exchanged virtual boson is purely space-like with 3-momentum $\mathbf{q}=(0,0,-Q)$, the incident quark carries momentum $Q / 2$ in the positive $Z$ direction, and the outgoing struck quark carries $\mathrm{Q} / 2$ in the negative $Z$ direction. A final state particle has a 4-momentum $p^{B}$ in this frame, and is assigned to the current region if $p_{Z}^{B}$ is negative, and to the target frame if $p_{Z}^{B}$ is positive. The advantage of this frame lies in the maximal separation of the outgoing parton from radiation associated with the incoming parton and the proton remnant, thus providing the optimal environment for the study of the fragmentation of the outgoing parton.

Event shape variables have been investigated in $e^{+} e^{-}$experiments and used to extract the strong coupling constant $\alpha_{s}\left(M_{Z}\right)$ independent of any jet algorithm, see eg ref. [23]. H1 have recently performed a similar analysis [24] in deep inelastic scattering in the current fragmentation region of the Breit frame. 
The event shape dependence on $Q$ (or energy dependence) can be due to the logarithmic change of the strong coupling constant $\alpha_{s}(Q) \propto 1 / \ln Q$, and/or power corrections (hadronisation effects) which are expected to behave like $1 / Q$. Recent theoretical developments suggest that $1 / Q$ corrections are not necessarily related to hadronisation, but may instead be a universal soft gluon phenomenon associated with the behaviour of the running coupling at small momentum scales [25, 26].

H1 have analysed a number of infrared safe (ie independent of the number of partons produced) event shape variables. Their definitions are given below, where the sums extend over all hadrons $h$ (being a calorimetric cluster in the detector or a parton in the QCD calculations) with four-momentum $p_{h}^{B}=\left\{E_{h}^{B}, \mathbf{p}_{h}^{B}\right\}$ The current hemisphere axis $\mathbf{n}=\{0,0,-1\}$ coincides with the virtual boson direction.

- Thrust $T_{c}$

$$
T_{c}=\max \frac{\sum_{h}\left|\mathbf{p}_{h}^{B} \cdot \mathbf{n}_{T}\right|}{\sum_{h}\left|\mathbf{p}_{h}^{B}\right|} \quad \mathbf{n}_{T} \equiv \text { thrust axis },
$$

- Thrust $T_{z}$

$$
T_{z}=\frac{\sum_{h}\left|\mathbf{p}_{h}^{B} \cdot \mathbf{n}\right|}{\sum_{h}\left|\mathbf{p}_{h}^{B}\right|}=\frac{\sum_{h}\left|\mathbf{p}_{z h}^{B}\right|}{\sum_{h}\left|\mathbf{p}_{h}^{B}\right|} \quad \mathbf{n} \equiv \text { hemisphere axis }
$$

- Jet Broadening $B_{c}$

$$
B_{c}=\frac{\sum_{h}\left|\mathbf{p}_{h}^{B} \times \mathbf{n}\right|}{2 \sum_{h}\left|\mathbf{p}_{h}^{B}\right|}=\frac{\sum_{h}\left|\mathbf{p}_{\perp h}^{B}\right|}{2 \sum_{h}\left|\mathbf{p}_{h}^{B}\right|} \quad \mathbf{n} \equiv \text { hemisphere axis }
$$

- Scaled Jet Mass $\rho_{c}$

$$
\rho_{c}=\frac{M^{2}}{Q^{2}}=\frac{\left(\sum_{h} p_{h}^{B}\right)^{2}}{Q^{2}} .
$$

A common characteristic of the mean event shape values $\left\langle 1-T_{c}\right\rangle,\left\langle 1-T_{z}\right\rangle,\left\langle B_{c}\right\rangle$ and $\left\langle\rho_{c}\right\rangle$ is the fact that they exhibit a clear decrease with rising $Q$, fig. 5. This is due to fact that the energy flow becomes more collimated along the event shape axis as $Q$ increases, a phenomenon also observed in $e^{+} e^{-}$annihilation experiments.

$\mathrm{H} 1$ showed by fitting to the data in fig. 5 all the event shape variables can be well described by just the first order power corrections $\propto 1 / Q$, without the need for any higher order corrections. The second order perturbative QCD parton predictions are also shown and their discrepancies with the data show that the power corrections are substantial at low values of $Q$, but become less important with increasing energy.

The analysis of the event shapes give results consistent with each other for $\bar{\alpha}_{0}$, the power correction parameter thus supporting the prediction of universality [25], and also gives consistent values of $\alpha_{s}\left(M_{Z}\right)$. The results of the fit are $\bar{\alpha}_{0}=0.491 \pm 0.003(\exp )_{-0.042}^{+0.079}$ (theory) for the power correction parameter and $\alpha_{s}\left(M_{Z}\right)=0.118 \pm 0.001$ (exp) ${ }_{-0.006}^{+0.007}$ (theory) for the strong coupling constant in the $\overline{\mathrm{MS}}$ scheme. These values are compatible with those extracted by $e^{+} e^{-}$ experiments 27] 

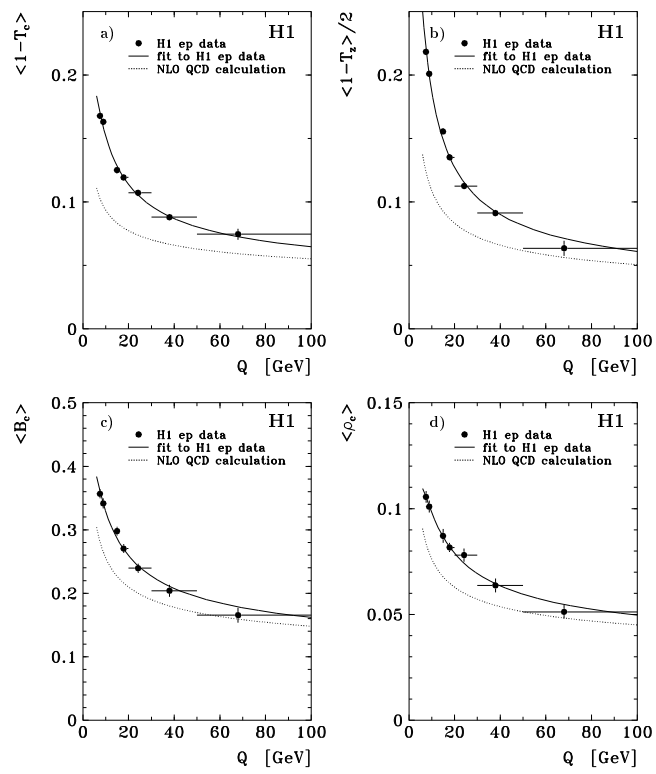

Figure 5: Mean event shape variables as a function of $Q$ for $\left.\left.\mathbf{a})\left\langle 1-T_{c}\right\rangle, \mathbf{b}\right)\left\langle 1-T_{z}\right\rangle / 2, \mathbf{c}\right)\left\langle B_{c}\right\rangle$, and $\mathbf{d})\left\langle\rho_{c}\right\rangle$. H1 DIS e p data (•, errors include statistics and systematics) are compared with $Q C D$ fits (-) and second order QCD calculations (...)

\section{$5 \quad$ Fragmentation Functions}

Fragmentation functions represent the probability for a parton to fragment into a particular hadron carrying a certain fraction of the parton's energy. Fragmentation functions incorporate the long distance, non-perturbative physics of the hadronization process in which the observed hadrons are formed from final state partons of the hard scattering process and, like structure functions, cannot be calculated in perturbative QCD, but can be evolved from a starting distribution at a defined energy scale. If the fragmentation functions are combined with the cross sections for the inclusive production of each parton type in the given physical process, predictions can be made for the scaled momentum, $x_{p}$, spectra of final state hadrons. Small $x_{p}$ fragmentation is significantly affected by the coherence (destructive interference) of soft gluons [28], whilst scaling violation of the fragmentation function at large $x_{p}$ allows a measurement of $\alpha_{s}$ [29].

In $e^{+} e^{-}$annihilation the two quarks are produced with equal and opposite momenta, $\pm \sqrt{s} / 2$. This can be compared with a quark struck from within the proton with outgoing momentum $-Q / 2$ in the Breit frame. In the direction of the struck quark (the current fragmentation region) the particle momentum spectra, $x_{p}=2 p^{B} / Q$, are expected to have a dependence on $Q$ similar to those observed in $e^{+} e^{-}$annihilation [30, 31, 32] at energy $\sqrt{s}=Q$.

The inclusive charged particle distributions [33, 34], $\left(1 / \sigma_{t o t}\right) d \sigma / d x_{p}$, are shown in figure 6 plotted in bins of fixed $x_{p}$ as a function of $Q^{2}$. For $Q^{2}>80 \mathrm{GeV}^{2}$ the distributions rise with $Q^{2}$ at low $x_{p}$ and fall-off at high $x_{p}$ and high $Q^{2}$. By measuring the amount of scaling violation one can ultimately measure the amount of parton radiation and thus determine $\alpha_{s}$. Below $Q^{2}=80 \mathrm{GeV}^{2}$ the fall off is due to depopulation of the current region.

The results can be compared to the next-to-leading order (NLO) QCD calculations, as implemented in CYCLOPS [35], of the charged particle inclusive distributions in the restricted region $Q^{2}>80 \mathrm{GeV}^{2}$ and $x_{p}>0.1$, where the theoretical uncertainties are small, unaffected by the hadron mass effects which are not included in the fragmentation function. This comparison 


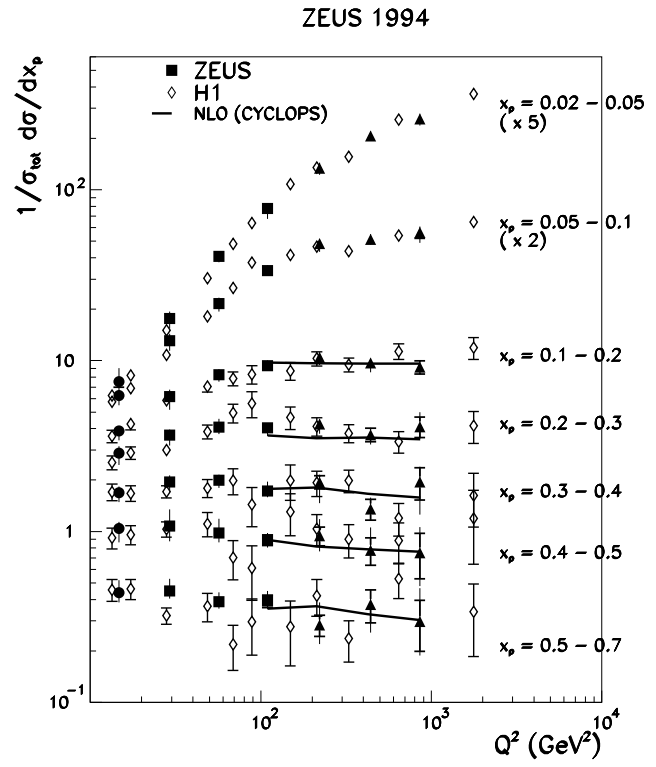

Figure 6: The inclusive charged particle distribution, $1 / \sigma_{\text {tot }} d \sigma / d x_{p}$, in the current fragmentation region of the Breit frame compared to the NLO calculation, CYCLOPS [35].

is shown in figure 6. The NLO calculation combines a full next-to-leading order matrix element with the $\mathrm{MRSA}^{\prime}$ parton densities (with a $\Lambda_{\mathrm{QCD}}=230 \mathrm{MeV}$ ) and NLO fragmentation functions derived by Binnewies et al. from fits to $e^{+} e^{-}$data [36]. The data and the NLO calculations are in good agreement, supporting the idea of universality of quark fragmentation.

The peak position of the $\xi=\ln \left(1 / x_{p}\right)$ distributions, $\xi_{\text {peak }}$, was evaluated. Figure 7 shows the distribution of $\xi_{\text {peak }}$ as a function of $Q$ for the HERA data [33, 37, 38] and of $\sqrt{s}$ for the $e^{+} e^{-}$data. Over the range shown the peak moves from $\simeq 1.5$ to 3.0 , equivalent to the position of the maximum of the corresponding momentum spectrum increasing from $\simeq 400$ to $900 \mathrm{MeV}$. The HERA data points are consistent with those from TASSO [39] data and a clear agreement in the rate of growth of the HERA points with the $e^{+} e^{-}$data [39, 40] is observed.

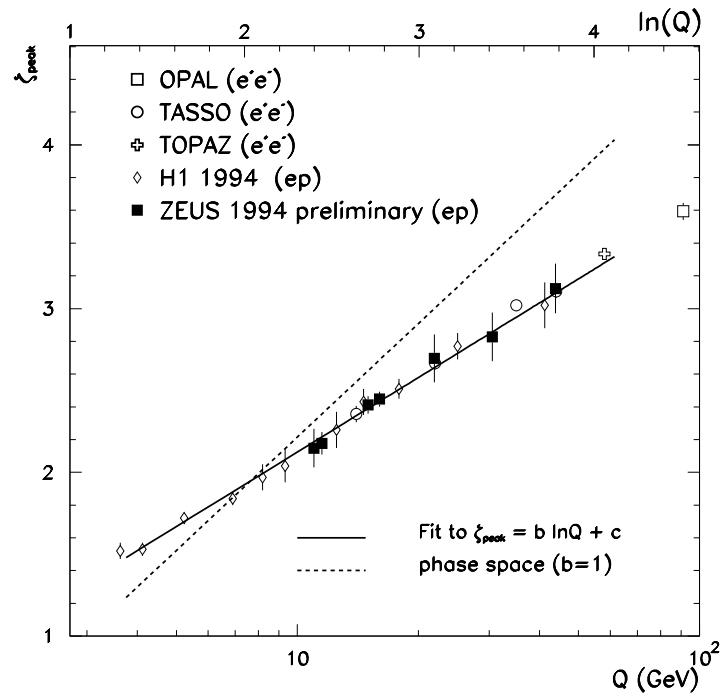

Figure 7: $\xi_{\text {peak }}$ as a function of $Q$. The HERA data are compared to results from OPAL, TASSO and TOPAZ. A straight line fit of the form $\xi_{\text {peak }}=b \ln (Q)+c$ to the ZEUS $\xi_{\text {peak }}$ values is indicated as well as the line corresponding to $b=1$, discussed in the text.

The increase of $\xi_{\text {peak }}$ can be approximated phenomenologically by the straight line fit $\xi_{\text {peak }}=$ $b \ln (Q)+c$ also shown in figure 7 . Also shown is the statistical fit to the data when $b=1$ which 
would be the case if the QCD cascade was of an incoherent nature, dominated by cylindrical phase space. (A discussion of phase space effects is given in 41].) In such a case, the logarithmic particle momentum spectrum would be peaked at a constant value of momentum, independent of $Q$. The observed gradient is clearly inconsistent with $b=1$ and therefore inconsistent with cylindrical phase space thus supporting the coherent nature of gluon radiation.

\section{BFKL versus DGLAP Evolution}

In the DGLAP parton evolution scheme [9] the parton cascade follows a strong ordering in transverse momentum $k_{T n}^{2} \gg k_{T n-1}^{2} \gg \ldots \gg k_{T 1}^{2}$, while there is only a soft (kinematical) ordering for the fractional momentum $x_{n}<x_{n-1}<\ldots<x_{1}$. However for low- $x$ at HERA the BFKL scheme [10] could well be the dominant scheme. In this scheme the cascade follows a strong ordering in fractional momentum $x_{n} \ll x_{n-1} \ll \ldots \ll x_{1}$, while there is no ordering in transverse momentum.

BFKL evolution can be enhanced by studying DIS events which contain an identified jet of longitudinal momentum fraction $x_{j e t}=p_{z}(j) / E_{\text {proton }}$ (in the proton direction) which is large compared to Bjorken $x$ [42]. By tagging a forward jet with $p_{T}(j) \simeq Q$ this allows minimal phase space for DGLAP evolution while the condition $x_{\text {jet }} \gg x$ leaves BFKL evolution active. This leads to the forward jet production cross section in BFKL dynamics being larger than that of the $\mathcal{O}\left(\alpha_{S}^{2}\right)$ QCD calculation with DGLAP evolution [43].

In Fig. 8, recent data from H1 44] and ZEUS [45] are compared with BFKL predictions 46] and fixed order QCD predictions as calculated with the MEPJET [17 program at NLO. The conditions $p_{T}(j) \simeq Q$ and $x_{j e t} \gg x$ are satisfied in the two experiments by slightly different selection cuts. H1 selects events with a forward jet of $p_{T}(j)>3.5 \mathrm{GeV}$ (in the angular region $\left.7^{\circ}<\theta(j)<20^{\circ}\right)$ with

$$
0.5<p_{T}(j)^{2} / Q^{2}<2, \quad x_{j e t} \simeq E_{\text {jet }} / E_{\text {proton }}>0.035 ;
$$

while ZEUS triggers on somewhat harder jets of $p_{T}(j)>5 \mathrm{GeV}$ and $\eta(j)<2.4$ with

$$
0.5<p_{T}(j)^{2} / Q^{2}<4, \quad x_{j e t}=p_{z}(j) / E_{\text {proton }}>0.035 .
$$

Fig. 8 shows that both experiments observe substantially more forward jet events than expected from NLO QCD. A very rough estimate of the uncertainty of the NLO calculation is provided by the two dotted lines, which correspond to variations by a factor 10 of the renormalisation and factorisation scales $\mu_{R}^{2}$ and $\mu_{F}^{2}$. A recent BFKL calculation (dashed lines) gives a better agreement with the data. The overall normalisation in this calculation is uncertain and the agreement may be fortuitous, indeed it should also be noted that both experiments observe more centrally produced dijet events than predicted by the NLO QCD calculations. Further careful investigation is necessary before claiming that BFKL is the mechanism for this enhanced forward jet production.

\section{Conclusions}

To understand the underlying QCD processes in DIS it is necessary to study the hadronic final state. At the current level of understanding, QCD works well and describes the HERA data. As the precision of the HERA data improves and the NLO QCD calculations become available the framework of QCD is being tested more thoroughly. As yet it is not possible to say conclusively whether the effects of BFKL dynamics are being observed in the HERA data, much theoretical 

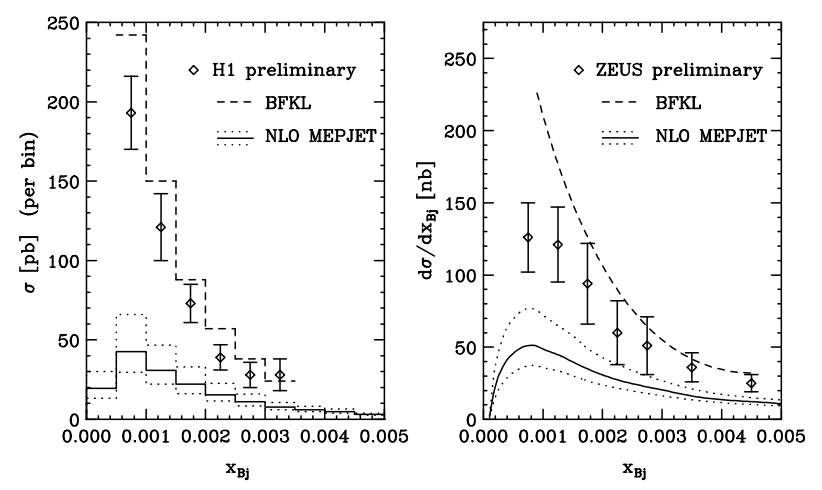

Figure 8: Forward jet cross section at HERA as a function of Bjorken $x$ within (a) the H1 [44] and (b) the ZEUS [45] acceptance cuts. The BFKL result of Bartels et al. [46] is shown as the dashed line. The solid and dotted line give the NLO MEPJET result and a measure for the uncertainity of NLO prediction through changes in the choice of scale.

and experimental work is in progress to define and measure variables that will allow a definitive statement.

\section{Acknowledgements}

I would like to thank the organising committee for inviting me to the school and providing such a pleasant and enjoyable environment.

\section{References}

[1] C. Alvisi et al., Nucl. Inst. Meth. A305 (1991) 30.

[2] N. Harnew et al., Nucl. Inst. Meth. A279 (1989) 290; B. Foster et al., Nucl. Phys. B (Proc. Suppl.) 32 (1993) 181; B. Foster et al., Nucl. Inst. Meth. A338 (1994) 254.

[3] M. Derrick et al., Nucl. Inst. Meth. A309 (1991) 77; A. Andresen et al., Nucl. Inst. Meth. A309 (1991) 101; A. Bernstein et al., Nucl. Inst. Meth. A336 (1993) 23.

[4] ZEUS Collab., M. Derrick et al., Z. Phys. C72 (1996) 399.

[5] J. Andruszków et al., DESY 92-066 (1992).

[6] ZEUS Collab., The ZEUS Detector, Status Report 1993, DESY 1993.

[7] H1 Collaboration, I. Abt et al., DESY preprint 93-103 (1993), Nucl. Instr. Meth. A386 (1997) 310 (Vol 1) and ibid. p.348 (Vol 2).

[8] S. Bentvelsen, J. Engelen and P. Kooijman, Proceedings of the 1991 Workshop on Physics at HERA, DESY Vol. 1 (1992) 23.

[9] G. Altarelli and G. Parisi, Nucl. Phys. 126 (1977) 297; V.N. Gribov and L.N. Lipatov, Sov. J. Nucl. Phys. 15 (1972) 438 and 675; Yu. L. Dokshitzer, Sov. Phys. JETP 46 (1977) 641.

[10] E.A. Kuraev, L.N. Lipatov and V.S. Fadin, Sov. Phys. JETP 45 (1977) 199; Y.Y. Balitsky and L.N. Lipatov, Sov. J. Nucl. Phys. 28 (1978) 282.

[11] JADE Collab., W. Bartel et al., Z. Phys. C33 (1986) 23;

JADE Collab., S. Bethke et al., Phys. Lett. B213 (1988) 235. 
[12] D. Graudenz, CERN-TH.7420/94 (1994), to appear in Comp. Phys. Comm.

[13] T. Brodkorb and E. Mirkes, Univ. of Wisconsin, MAD/PH/821 (1994).

[14] H1 Collab., T. Ahmed et al., Phys. Lett. B346 (1995) 415.

[15] ZEUS Collab., M. Derrick et al., Phys. Lett. B363 (1995) 201.

[16] Particle Data Group, R.M. Barnett et al., Phys. Rev. D54 (1996).

[17] E. Mirkes and D. Zeppenfeld, Phys. Lett. B380 (1996) 205.

[18] S. Catani and M. Seymour, Nucl. Phys. B 485 (1997) 291 and hep-ph/9605323;

[19] S. Catani, Y. Dokshitzer and B. Webber, Phys. Lett. B285 (1992) 291.

[20] B. R. Webber, J. Phys. G19 (1993) 1567.

[21] T. Trefzger, Proc. of the Int. Workshop on DIS and Related Phenomena, ed. G. D'Agostini and A. Nigro, Rome, 1996.

[22] R.P. Feynman, "Photon-Hadron Interactions", Benjamin, N.Y. (1972).

[23] S. Bethke, Proceedings QCD 94, Montpellier, ed. S Narison, Nucl. Phys. B (Proc. Suppl.) 39 B, C (1995), p. 198.

[24] H1 collab., C. Aloff et al., DESY-97-098. hep-ex/9706002

[25] B.R. Webber, Proceedings Workshop on Deep Inelastic Scattering and QCD, Paris (1995), eds. J.F. Laporte and Y. Sirois, p. 115; M. Dasgupta and B.R. Webber, preprint Cavendish-HEP-96/5 and hep$\mathrm{ph} / 9704297$.

[26] Yu.L. Dokshitzer and B.R. Webber, Phys. Lett. B 352 (1995) 451; Z. Kunszt, P. Nason, G. Marchesini and B.R. Webber, Z Physics at LEP 1, eds. G. Altarelli, R. Kleiss and C. Verzegnassi, CERN 89-08, vol. 1, p. 373.

[27] PLUTO Collaboration, Ch. Berger et al., Z. Phys. C 12 (1982) 297; Mark II Collaboration, A. Peterson et al., Phys. Rev. D 37 (1988) 1; TASSO Collaboration, W. Braunschweig et al., Z. Phys. C 45 (1989) 11 and Z. Phys. C 47 (1990) 187; AMY Collaboration, Y.K. Li et al., Phys. Rev. D 41 (1990) 2675; DELPHI Collaboration, P. Abreu et al., Z. Phys. C 73 (1997) 229.

[28] Yu. Dokshitzer, V. Khoze, A. Mueller and S. Troyan, "Basics of Perturbative QCD", Editions Frontières, Gif-sur-Yvette, France (1991).

[29] G. Altarelli et al., Nucl. Phys. B160 (1979) 301; P. Nason and B. R. Webber, Nucl. Phys. B421 (1994) 473.

[30] Yu. Dokshitzer et al., Rev. Mod. Phys. 60 (1988) 373.

[31] A. V. Anisovich et al., Il Nuovo Cimento, A106 (1993) 547.

[32] K. Charchuła, J. Phys. G19 (1993) 1587.

[33] H1 collab., C. Adloff et al., DESY-97-158 [hep-ex/9707005 fig. 2 amended 15th August 1997]

[34] ZEUS collab., J. Breitweg et al., DESY-97-183.

[35] D. Graudenz, CERN-TH/96-52; D. Graudenz, CYCLOPS program and private communication.

[36] J. Binnewies et al., Z Phys C65 (1995) 471.

[37] ZEUS Collaboration, M. Derrick et al., Z. Phys. C67 (1995) 93.

[38] H1 Collaboration, S. Aid et al., Nucl. Phys. 445B (1995) 3.

[39] TASSO Collab., W. Braunschweig et al., Z. Phys. C47 (1990) 187; TASSO Collab., W. Braunschweig et al., Z. Phys. C22 (1984) 307.

[40] OPAL Collab., M. Akrway et al., Phys. Lett. B247 (1990) 617. TOPAZ Collab., R. Itoh et al., Phys. Lett. B345 (1995) 335.

[41] E.R. Boudinov, P.V. Chliapnikov and V.A. Uvarov, Phys. Lett. B309 (1993) 210.

[42] A.H. Mueller, Nucl. Phys. B (Proc. Suppl.) 18C (1990) 125; J. Phys. G17 (1991) 1443; J. Kwiecinski, A.D. Martin and P.J. Sutton, Phys. Rev. D46 (1992) 921; W.K. Tang, Phys. Lett. B278 (1992) 363.

[43] E. Mirkes and D. Zeppenfeld, Phys. Rev. Lett. 78 (1997) 428 hep-ph/9609231]. 
[44] M. Wobisch, to appear in proceedings of DIS97 (and references therein).

[45] S. Wölfle, to appear in proceedings of DIS97

[46] J. Bartels et al., Phys. Lett. B384 (1996) 300 hep-ph/9604272. 